\title{
Software development of DC magnetic bias calculation in the power system
}

\author{
Yongjun $\mathrm{Yu}^{1, \mathrm{a}}$, Xiaoxiao $\mathrm{Qi}^{1}$, Peilun Zhang ${ }^{2, \mathrm{~b}}$, Bing Sun ${ }^{1}$, Yanhui Qin ${ }^{1}$, Zhiyuan \\ $\mathrm{Hou}^{3}$ and Liang Wang ${ }^{3}$ \\ ${ }^{1}$ Electric Power Research Institute of Xinjiang Power Grid Corporation, Urumchi 830000, China \\ ${ }^{2}$ School of Electrical Engineering, Wuhan University, Wuhan 430072, China \\ ${ }^{3}$ Anhui ZGD Electric Power Technology Co.Ltd, Hefei 230088, China. \\ ayujyuj@vip.sina.com, ${ }^{b}$ zhangpl@whu.edu.cn.
}

Keywords: DC magnetic bias, AC power grid, DC network information software.

\begin{abstract}
The safe and reliable operation of power system has been severely threatened by frequent DC magnetic bias when the applied DC transmission system is operating in a single-polar ground loop mode or a bipolar asymmetric mode. Based on SQL SERVER 2012, this paper sets up an AC-grid DC network information database platform, which is aimed at DC bias problems. The paper also focuses on the respective development of simulation modules for ground, transmission lines, transformers, substations as well as the comprehensive one for DC current calculation in AC power grid. Then Visual Studio 2010 is applied to develop power system DC bias calculation software. The accuracy of AC-grid DC network simulation modules is finally proved by means of practical examples and comparison to other power system modulation software.
\end{abstract}

\section{Introduction}

DC transmission in our country has recently undergone a rapid development. At the moment, several extra-high voltage DC transmission systems, such as Ge-Shang, San-Guang, Gui-Guang, Tian-Guang, San-Chang and so on, have been put into operation. With the development of Southwest Hydropower Exploitation and West to East Power Transmission Project, another $\pm 800 \mathrm{kV}$ DC transmission systems will be applied. There have been several DC bias occasions when the applied DC transmission system is operating in a single-polar ground loop mode or a bipolar asymmetric mode.

Traditional methods fail to reflect the complexity of special distribution. For example, some software such as PSCAD, POWER WORLD, PSSE and so forth are not able to input geographic locations of overground substations and they can't modulate underground resistivity distribution either. Nor can traditional methods modulate electric field effect of currents injecting into ground. Given our rapid development of DC transmission project and the increasing hazard of transformer DC bias in AC power grid, it is extremely imperative to develop an AC-grid DC distribution software.

Based on SQL SERVER 2012, this paper sets up an AC-grid DC network information database platform, which is aimed at DC bias problems. Then Visual Studio 2010 is applied to develop power system DC bias calculation software.

\section{The development of AC-grid DC network modules}

The development of ground simulation module. In terms of grounding, horizonal multi-layer soil is generally applied to simulate heterogeneous soil. With the application of separation of variables method, the potential function of the $\boldsymbol{i}$ th layer is expressed as

$$
\phi_{i}^{m}(r, z)=\frac{\rho_{i} I}{4 \pi} \int_{0}^{+\infty}\left[\delta(m-i) e^{-\lambda\left|z-h_{0}\right|}+A_{i}^{m}(\lambda) e^{-\lambda\left(z-h_{0}\right)}+B_{i}^{m}(\lambda) e^{\lambda\left(z-h_{0}\right)}\right] J_{0}(\lambda r) d \lambda
$$


Where $\phi_{i}^{m}(\boldsymbol{r}, \mathbf{z})$ stands for the Green's function of the $i$ th layer soil when the source is in the $\boldsymbol{m}$ th layer; $\lambda$ denotes an integral variable introduced in Bessel function transform; $\boldsymbol{J}_{0}$ means the first type 0 order Bessel function; $\boldsymbol{A}_{i}^{m}(\lambda)$ and $\boldsymbol{B}_{i}^{m}(\lambda)$ are coefficients to be worked out.

Through software package, the electric field calculation of horizonal multi-layer soil is packaged in the soil information sort of the software. The values of soil layer, layer resistivity and depth can be altered by inputting the information of horizonal multi-layer soil. And field calculation is fulfilled by means of soil information sort modules packaged in the software.

Development of transmission line module. The line module is in fact three resistive branches connected to the three-phase substation bus. There are two key parameters: DC resistance and its connecting way. The estimated DC resistance of the lines can be expressed as:

$$
R_{L}=\frac{\rho D}{S}
$$

Where $\mathbf{R}_{\mathbf{L}}$ stands for the single phase line resistance $(\Omega)$; $\boldsymbol{\rho}$ stands for the resistivity coefficient of the line material; $\mathbf{S}$ stands for the sectional area of a single phase; $\mathbf{D}$ means the line length.

Two relevant parameters, the name of the transmission line as well as the circuit number, are required for the simulation modeling. In terms of each transmission line circuit, some other parameters are required: its voltage grade, starting and ending bus, resistivity, length and sectional area.

Development of transformer module. A transformer model includes the winding type, the DC winding resistance and the transformer bus. The delta connection can be neglected due to its zero-sequence open circuit. As a result, a Y/D double winding transformer can be perceived as a Y single winding one in a DC distribution model. Similarly, a Y/Y/D triple winding transformer can be seen as a $\mathrm{Y} / \mathrm{Y}$ double winding one.

In a DC current distribution calculation, whether it is a self-coupling transformer has to be determined. In the case of a double winding transformer, its neutral might be disconnected to the grounding network or it might also be connected through a small reactance. Sometimes it needs to be connected in series with a resistance or capacitor due to the DC bias suppression.

Development of substation module. Of all DC models, a substation model is the most complicated, which in detail can be classified as overground part and underground part. Figure 1 shows a $500 \mathrm{kV}$ substation equivalent model in a segmented operating mode with a median voltage side bus of a double self-coupling transformer.

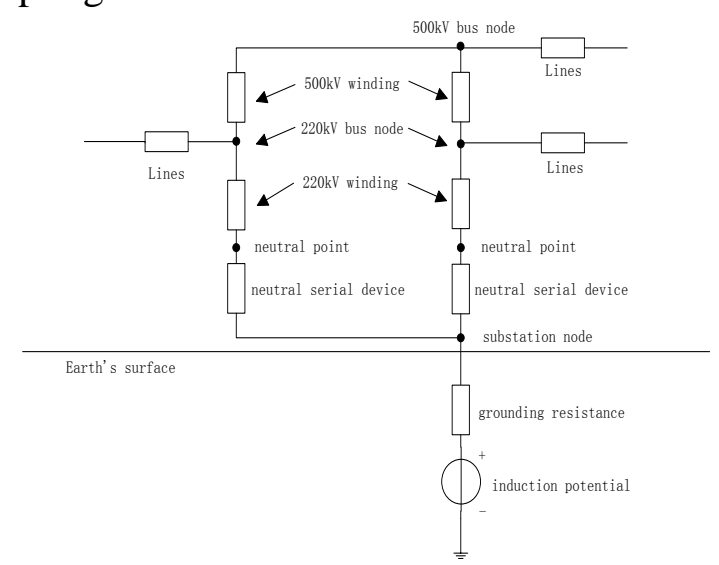

Fig.1. DC model of AC power grid

Apart from internal transformer modeling, bus modeling and neutral modeling, some other relevant parameters are needed when building a substation simulation model: substation's name, its latitude and longitude (degree), voltage grade (V) and grounding resistance $(\Omega)$.

Development of the comprehensive simulation module. If an AC grid has in all m substations, b bus nodes, $\mathrm{n}$ independent neutrals, according to nodal voltage method. We have

$\mathbf{Y V}=\mathbf{J}$ 
Where $\boldsymbol{V}$ stands for the line nodal voltage vector, $\mathbf{Y}$ stands for the conductance matrix of grid node, $\boldsymbol{J}$ stands for the line vector of the current injecting into the grid node.

\section{$\mathbf{Y}=\mathbf{H}^{\mathrm{T}} \mathbf{G}+\mathbf{Q}$}

Where $\boldsymbol{H}$ stands for the incidence matrix of substation nodes and all other nodes, $\mathbf{H}^{\mathbf{T}}$ is the transposition of $\boldsymbol{H}, \mathbf{H}_{\boldsymbol{m} \times(\boldsymbol{m}+\boldsymbol{n}+\boldsymbol{b})}=\left[\mathbf{E}_{\boldsymbol{m}} \mathbf{0}_{\boldsymbol{m} \times \boldsymbol{n}} \mathbf{0}_{\boldsymbol{m} \times \boldsymbol{b}}\right], \mathbf{E}_{\mathbf{m}}$ is an $m$ th order unit matrix, $\mathbf{G}$ is the grounding conductance matrix of the substation, $\mathbf{G}=\mathbf{R}^{-1}, \mathbf{R}=\operatorname{diag}\left(\boldsymbol{R}_{G 1}, \boldsymbol{R}_{G 2}, \ldots, \boldsymbol{R}_{G m}\right), \boldsymbol{R}_{G i}$ is the DC grounding resistance of the $\boldsymbol{i}$ th substation $(\Omega)$.

The potential of the substation ground surface is

$$
\mathbf{P}=\mathbf{M I}_{\mathbf{D}}+\mathbf{N I}_{\mathrm{A}}
$$

Where $\boldsymbol{M}$ stands for the mutual resistance matrix between the DC pole and the substation, $\mathbf{N}$ stands for the mutual impedance matrix of each substation (excluding self-reaction), $\boldsymbol{I}_{\boldsymbol{D}}$ is the grounding current of the DC pole, and $\boldsymbol{I}_{\boldsymbol{A}}$ is the DC current of the substation neutral. For a multi-level soil model, both mutual impedance between substation and grounding pole and between each substation can be obtained through equation 1.

The substation grounding voltage

$\mathbf{V}_{\mathbf{A}}=\mathbf{H V}$

The DC current injecting into the substation grounding network is

$$
\mathbf{I}_{\mathrm{A}}=\mathbf{G}\left(\mathbf{V}_{\mathrm{A}}-\mathbf{P}\right)
$$

According to the simultaneous equations, the neutral DC current is

$\mathbf{I}_{\mathrm{A}}=(\mathbf{R}-\mathbf{Z N})^{-1} \mathbf{Z M I}_{\mathrm{D}}$

Where $\mathbf{Z}=\mathbf{H} \mathbf{Y}^{-\mathbf{1}} \mathbf{H}^{\mathbf{T}} \mathbf{G}$-E. In a DC distribution model, if the neutral is disconnected, then $\mathbf{Z}(\mathbf{i}, \mathbf{i})=\mathbf{0}$, $I_{A}(\mathbf{i})=\mathbf{0}$.

According to the above thought, based on SQL SERVER 2012, this paper sets up an AC-grid DC network information database platform, which is aimed at DC bias problems. Then Visual Studio 2010 is applied to develop power system DC bias calculation software.

\section{The development of AC-grid DC network modules}

With the aim of verifying the effectiveness of the simulation method with this software, the method in this paper and MATLAB SIMULINK, PSCAD and ATP software are respectively applied to calculate the current distribution on Hami substation and Tianshan HVDC converter station, in the case of Hami--Zhengzhou $\pm 800 \mathrm{kV}$ UHVDC Jijitai DC pole 500A current injecting into ground.

The AC grid simulation model is constructed in the DC current AC grid distributed computing software, which contains only two substations, a line and a converter station earth electrode.

The main parameters of Hami, Tianshan station and the lines between them are in the following tables.

Table 1 Main parameters of two substations

\begin{tabular}{ccccc}
\hline \multirow{2}{*}{ Substation } & $\begin{array}{c}\text { Voltage } \\
\text { rating } \\
(\mathrm{kV})\end{array}$ & $\begin{array}{c}\text { Ground } \\
\text { resistance } \\
(\Omega)\end{array}$ & $\begin{array}{c}\text { DC resistance of } \\
\text { High voltage } \\
\text { winding }\end{array}$ & $\begin{array}{c}\text { Medium voltage } \\
\text { winding }\end{array}$ \\
\hline Hami & 750 & 0.2 & 0.1664 & 0.007935 \\
Tianshan & 750 & 0.2 & 0.1475 & 0.00805 \\
\hline
\end{tabular}

Table 2 Main parameters of lines

\begin{tabular}{cccc}
\hline Circuit loop count & $\begin{array}{c}\text { Loop resistance } \\
\operatorname{rate}\left(\Omega \cdot \mathrm{mm}^{2} / \mathrm{km}\right)\end{array}$ & Length $(\mathrm{km})$ & Cross-sectional area $\left(\mathrm{mm}^{2}\right)$ \\
\hline 2 & 30.8952 & 70 & 2400 \\
\hline
\end{tabular}

With this development software simulated, the neutral-point voltage of Hami substation and Tianshan converter station are $-9.954713 \mathrm{~V}$ and $-9.05796 \mathrm{~V}$ respectively. The DC resistance of a 
single loop circuit is $30.8952 * 70 / 2400=0.9011 \Omega$. The double loop circuit counterpart is $0.9011 / 2=0.4506 \Omega$. The results of calculation are in the following tables.

Table 3 Results of calculation by different power system softwares

\begin{tabular}{ccccc}
\hline $\begin{array}{c}\text { Power system } \\
\text { softwares }\end{array}$ & $\begin{array}{c}\text { AC-grid DC } \\
\text { network } \\
\text { simulation } \\
\text { modules }\end{array}$ & Matlab Simulink & PSCAD & ATP \\
\hline $\begin{array}{c}\text { Earth current (A) } \\
\text { Relative error }\end{array}$ & 3.51918 & 3.51898 & 3.5200379 & 3.51898 \\
\hline
\end{tabular}

Through comparison and analysis of Matlab Simulink, PSCAD and ATP, the biggest relative error is $0.006 \%$, while the lowest $0.02437 \%$, which denotes the accuracy of the AC-grid DC current network simulation modules.

\section{Summary}

The project develops ground electric field simulation modules, transmission line modules, transformer modules and substation modules.

Based on SQL SERVER 2012, this paper sets up an AC-grid DC network information database platform, which is aimed at DC bias problems. Then Visual Studio 2010 is applied to develop power system DC bias calculation softwares.

The accuracy of AC-grid DC network simulation modules is finally proved by means of practical examples and comparison to other power system modulation softwares.

\section{References}

[1] A. F. Otero, J. Cidras, J. L. Alamo. Frequency-dependent grounding system calculation by means of a conventional nodal analysis technique [J]. IEEE Trans. Power Delivery, 1999, 14(3), 873-878.

[2] Zhu Yi-ying, Jiang Wei-ping, Zeng Zhao-hua. Studying on measures of restraining DC current through transformer neutrals [J].Proceedings of the CSEE, 2005, 25(13), 3-7.

[3] Ma Zhi-qiang, Li Xiao-lin, Zhong Ding-zhu. Network algorithm to analyze effects of DC transmission ground current on AC system[J].Guangdong Electric Power, 2005, 18(12), 4-8.

[4] Cao Zhao jun, He Junjia, Ye Huisheng. Method for the Calculation of DC Current Distribution in AC System when HVDC Operating in Ground-return Mode.High Voltage Engineering, 2006, 32(10), 82-84.

[5] Zhang Bo, Zhao Jie, Zeng Rong. Estimation of DC current distribution in AC power system caused by HVDC transmission system in ground return status. Proceedings of the CSEE, 2006, 26(13), 84-88.

[6] Zeng Rong, Zhao Jie, Shang Chun. Measures to Restrain the Neutral Current of the AC Transformer in HVDC Ground Return System [J].High Voltage Engineering, 2009, 35(04), 919-925.

[7] Zeng Rong, Zhang Bo, Zhao Jie, et al. Influence and Characteristics Analysis of Effect of the HVDC Ground Return Current on AC System[J]. High Voltage Engineering, 2009, 35(3), 678-682.

[8] Cao Lin, Zeng Rong, Zhao Jie. Study on the Influence of HVDC System Grounding Electrode Current on Power Transformer Operation [J]. High Voltage Apparatus, 2006, 42(5), 346-348.

[9] Pan Zhuohong, Zhang Lu, Tan Bo, et al. Simulation and Analysis of HVDC Earth-return Current's Distribution in AC Power Grid [J]. Automation of Electric Power Systems, 2011, 35(21). 